\title{
Algebraic dimension of complex nilmanifolds
}

\author{
Anna Fino, Gueo Grantcharov, Misha Verbitsky 1
}

\begin{abstract}
Let $M$ be a complex nilmanifold, that is, a compact quotient of a nilpotent Lie group endowed with an invariant complex structure by a discrete lattice. A holomorphic differential on $M$ is a closed, holomorphic 1-form. We show that $a(M) \leqslant k$, where $a(M)$ is the algebraic dimension $a(M)$ (i.e. the transcendence degree of the field of meromorphic functions) and $k$ is the dimension of the space of holomorphic differentials. We prove a similar result about meromorphic maps to Kahler manifolds.
\end{abstract}

\section{Contents}

1 Introduction 1 1.1 Nilmanifolds: definition and basic properties . . . . . . . . 1 1.2 Algebraic dimension and holomorphic differentials . . . . . . 3

2 The averaging formalism 6

3 Positive currents on nilmanifolds

3.1 Holomorphic differentials . . . . . . . . . . . . . . 7 G

3.2 Positive $(1,1)$-forms on a Lie algebra . . . . . . . . . . 8

3.3 Averaging the positive currents ............. 11

4 Examples $\quad 12$

4.1 Algebraic dimension of complex 2-tori . . . . . . . . . . 12

4.23 -dimensional complex nilmanifolds . . . . . . . . . . 14

\section{Introduction}

\subsection{Nilmanifolds: definition and basic properties}

A nilmanifold is a compact manifold equipped with a transitive action of a nilpotent Lie group. As shown by Malcev ([M]), every nilmanifold can be obtained as a quotient of a nilpotent Lie group $G$ by a discrete lattice

\footnotetext{
${ }^{1}$ Anna Fino is partially supported by PRIN, FIRB and GNSAGA (Indam), Gueo Grantcharov is supported by a grant from the Simons Foundation (\#246184), and Misha Verbitsky is partially supported by RSCF grant 14-21-00053 within AG Laboratory NRUHSE.
} 
$\Gamma$. Moreover, the group $G$ can be obtained as so-called Malcev completion of $\Gamma$, that is, as a product of exponents of formal logarithms of elements $\Gamma$. Therefore, any nilmanifold is uniquely determined by its fundamental group, which is a discrete nilpotent torsion-free group, and any such group uniquely determines a nilmanifold.

Since the work of Thurston $([\mathrm{T}])$, geometric structures on nilmanifolds are used to provide many interesting examples (and counterexamples) in complex and symplectic geometry. It was Thurston who realized that the Kodaira surface (also known as a Kodaira-Thurston surface) is symplectic, but does not admit any Kähler structure. In this way Thurston obtained a counterexample to a result stated by H. Guggenheimer (G] in 1951. Guggenheimer claimed that the Hodge decomposition is true for compact symplectic manifolds, but for symplectic nilmanifolds this is usually false.

Before 1990-ies, a "complex nilmanifold" meant a compact quotient of a complex nilpotent Lie group by a discrete, co-compact subgroup. The first non-trivial example is given by so-called Iwasawa manifold ( $\mathrm{FG}$ ) which is obtained as a quotient of the 3-dimensonal Lie group of upper triangular 3 by 3 matrices by a discrete co-compact subgroup, for example the group of upper triangular matrices with coefficients in $\mathbb{Z}[\sqrt{-1}]$.

Starting from late 1980-ies, a "complex nilmanifold" means a quotient of a real nilpotent Lie group equipped with a left-invariant complex structure by the left action of a discrete, co-compact subgroup ([CFG2]). This is the notion we are going to use in this paper. This definition is much more general, indeed, left-invariant complex structures are found on many even-dimensional nilpotent Lie groups which are not complex. The complex structure on a Kodaira surface is one of such examples.

Complex structures on a nilmanifold have a very neat algebraic characterization. Let $G$ be a real nilpotent Lie group, and $\mathfrak{g}$ is Lie algebra. By Newlander-Nirenberg theorem, a complex structure on $G$ is the same as a sub-bundle $T^{1,0} G \subset T G \otimes_{\mathbb{R}} \mathbb{C}$ such that $\left[T^{1,0} G, T^{1,0} G\right] \subset T^{1,0} G$ and $T^{1,0} G \oplus \overline{T^{1,0} G}=T G \otimes_{\mathbb{R}} \mathbb{C}$. The left-invariant sub-bundles in $T^{1,0} G$ are the same as subspaces $W \subset \mathfrak{g} \otimes_{\mathbb{R}} \mathbb{C}$, and the condition $\left[T^{1,0} G, T^{1,0} G\right] \subset T^{1,0} G$ is equivalent to $[W, W] \subset W$. Therefore, left-invariant complex structures on $G$ are the same as complex sub-algebras $\mathfrak{g}^{1,0} \subset \mathfrak{g} \otimes_{\mathbb{R}} \mathbb{C}$ satisfying $\mathfrak{g}^{1,0} \oplus \overline{\mathfrak{g}^{1,0}}=$ $\mathfrak{g} \otimes_{\mathbb{R}} \mathbb{C}$.

A real nilmanifold is obtained as an iterated fibration with fibers which are compact tori. It is natural to ask if any complex nilmanifold can be obtained as an iterated fibration with fibers which are complex tori. The answer is negative: see e.g. $[\mathbf{R}$.

However, a weaker statement is still true. If we replace fibrations of nilmanifolds by homomorphisms of their Lie algebras, it is possible to construct a homomorphism $\psi: \mathfrak{g} \longrightarrow \mathfrak{a}$ to a complex abelian Lie algebra compatible 
with a complex structure. Since $\mathfrak{a}$ is abelian, ker $\psi$ necessarily contains the commutator $[\mathfrak{g}, \mathfrak{g}]$. Since it is complex, $\operatorname{ker} \psi$ contains $[\mathfrak{g}, \mathfrak{g}]+I[\mathfrak{g}, \mathfrak{g}]$.

The quotient algebra $\mathfrak{g} /[\mathfrak{g}, \mathfrak{g}]+I[\mathfrak{g}, \mathfrak{g}]$ is called the algebra of holomorphic differentials on $G$, denoted by $\mathfrak{H}^{1}(M)$. Its dimension is always positive ([S] $)$.

In this paper, we study algebraic objects (such as meromorphic functions) on complex nilmanifolds. We prove the following theorem.

Theorem 1.1: Let $M=G / \Gamma$ be a complex nilmanifold, and $\Sigma$ be a a foliation obtained by left translates of $[\mathfrak{g}, \mathfrak{g}]+I[\mathfrak{g}, \mathfrak{g}]$, where $I$ is the complex structure operator, and $\mathfrak{g}=T_{e} G$ the Lie algebra of $G$. Then all meromorphic functions on $M$ are constant on the leaves of $\Sigma$.

Proof: See Subsection 3.3 .

\subsection{Algebraic dimension and holomorphic differentials}

Recall that a positive closed $(1,1)$-current $T$ on a complex manifold is said to have analytic singularities (see [Bouck]) if locally $T=\theta+d d^{c} \varphi$ for a smooth form $\theta$ and a plurisubharmonic function $\varphi=c \log \left(\left|f_{1}\right|^{2}+\ldots+\left|f_{n}\right|^{2}\right)$ where $f_{1}, \ldots f_{n}$ are analytic functions and $c$ a constant. Such currents have decomposition into absolutely continuous and singular part, where the absolutely continuous part is positive and closed.

Definition 1.2: Let $M$ be a complex manifold. The Kähler rank $k(M)$ of $M$ is the maximal rank of the absolutely continuous part of a positive, closed (1,1)-current on $M$ with analytic singularities.

Definition 1.3: The algebraic dimension $a(M)$ of a complex manifold is the transcendence degree of its field of meromorphic functions.

Let $X$ be a complex manifold, and $\varphi: X \rightarrow \mathbb{C}^{n}$ a meromorphic map defined by generators of the field of meromorphic functions. An algebraic reduction of $X$ ([Ca81, [Ue75]) is a compactification of $\varphi(X)$ in $\mathbb{C} P^{n} \supset \mathbb{C}^{n}$. It is known to be a compact, algebraic variety ([Ca81, [Ue75]).

We should note that the map $\varphi$ is defined for more general spaces $X$. For smooth manifolds we'll use the following [Pet, Definition-Theorem 6.5].

Definition 1.4: Let $M$ be a compact complex manifold. Then there exists 
a smooth projective manifold $X$, a rational map $\varphi: M \rightarrow X$ and a diagram

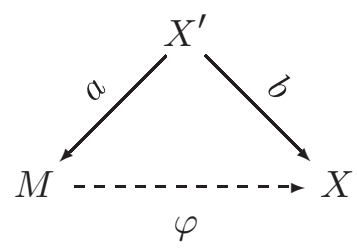

where $X^{\prime}$ is smooth and the top two arrows are proper holomorphic maps with $a$ a proper bimeromorphic modification, such that the corresponding fields of meromorphic functions coincide: $\operatorname{Mer}(M)=\operatorname{Mer}(X)$. We call the map $\varphi: M \rightarrow X$ algebraic reduction of $M$.

Definition 1.5: Let $\eta$ be a positive $(1,1)$-form on $X$. The current $T_{\eta}$ is defined as $a_{*} b^{*} \eta$; since pushforward of a form is a current, it is a current, and not a form. Since $a$ is one-to-one everywhere, except on an analytic set $E \subset X^{\prime}$, the current $a_{\star} b^{*} \eta$ is smooth outside of $E$. Note also that the positivity and closedness are preserved, as well as the rank in a general point. We call $T_{\eta}$ the current induced by $\eta$ on $M$. It is easy to check that $T_{\eta}$ has analytic singularities if $\eta$ is closed and positive.

Claim 1.6: Let $M$ be a complex variety. Then the algebraic dimension is bounded by the Kähler rank:

$$
a(M) \leqslant k(M) .
$$

Proof: Let $\varphi: M \rightarrow X$ be the algebraic reduction map. Pullback of a Kähler form from $X$ to $M$ is a current of $\operatorname{rank} \operatorname{dim} X$ at all points where it is absolutely continuous.

We are going to estimate $a(M)$ in terms of holomorphic differentials on $M$.

Definition 1.7: A holomorphic differential on a compact complex manifold is a closed, holomorphic 1-form.

Theorem 1.8: Let $M$ be a complex nilmanifold, $\mathfrak{H}^{1}(M)$ the space of holomorphic differentials on $M$, and $a(M)$ its algebraic dimension. Then

$$
a(M) \leqslant \operatorname{dim} \mathfrak{H}^{1}(M) .
$$

Proof: See Subsection 3.3 . 
Remark 1.9: The same estimate holds for complex parallelizable manifolds; see [W, Proposition 3.16.1]. Moreover, Theorem 1.1 is also true in this situation: all meromorphic functions are constant on the fibers of the Albanese map.

This result is implied by Definition 1.4 and the following expression of the Kähler rank.

Theorem 1.10: Let $M$ be a complex nilmanifold, $\mathfrak{H}^{1}(M)$ the space holomorphic differentials on $M$, and $k(M)$ its Kähler rank. Then $k(M)=$ $\operatorname{dim} \mathfrak{H}^{1}(M)$.

Proof: Consider the projection $\mathfrak{g} \longrightarrow \mathfrak{a}$, where $\mathfrak{a}=\frac{\mathfrak{g}}{[\mathfrak{g}, \mathfrak{g}]+I([\mathfrak{g}, \mathfrak{g}])}$. Since $[\mathfrak{g}, \mathfrak{g}]+I([\mathfrak{g}, \mathfrak{g}])$ is $I$-invariant, $a$ has a complex structure and this map is compatible with it. Consider the Chevalley differential $d$ on the Lie algebras of $\mathfrak{g}$ and $\mathfrak{a}$. Since $\mathfrak{a}$ is an abelian algebra, any 2-form on $\mathfrak{a}$ is closed (and gives a closed 2-form on the corresponding Lie group). Taking a positive definite Hermitian form, we obtain a positive current of rank $\operatorname{dim} \mathfrak{a}=\mathfrak{H}^{1}(M)$ on $M$. There are no currents with greater rank by Proposition 3.15.

The same argument implies the following useful corollary.

Corollary 1.11: Let $M$ be a complex nilmanifold, $\mathfrak{g}$ the Lie algebra of the corresponding Lie group, and $\mathfrak{h}:=[\mathfrak{g}, \mathfrak{g}]+I([\mathfrak{g}, \mathfrak{g}])$ the algebra constructed as above. Denote by $\mathfrak{h}_{1}$ a smallest $I$-invariant rational subspace of $\mathfrak{g}$ containing $\mathfrak{h}$. Let $T$ be a complex torus obtained as quotient of $\mathfrak{g} / \mathfrak{h}_{1}$ by its integer lattice. Consider the natural holomorphic projection $\Psi: M \longrightarrow T$. Then any meromorphic map to a Kähler manifold is factorized through $\Psi$.

Proof: Let $\psi: M \rightarrow X$ be a meromorphic map to a Kähler manifold $(X, \omega)$. For general $x \in X$, the zero space of the positive closed current $\psi^{*} \omega$ contains $\mathfrak{h}$, hence the fibers $F_{x}:=\psi^{-1}(x)$ are tangent to $\mathfrak{h}$. The smallest compact complex subvariety of $M$ containing a leaf of the foliation associated with $\mathfrak{h}$ is the corresponding leaf of $\mathfrak{h}_{1}$. Passing to the closures of the leaves of $\mathfrak{h}$, we obtain that $F_{x}$ contain leaves of $\mathfrak{h}_{1}$. However, $T$ is the leaf space of $\mathfrak{h}_{1}$.

Remark 1.12: For a general compact complex manifold $X$, Albanese variety $\operatorname{Alb}(X)$ is defined as the quotient of the dual space of the space of holomorphic differentials $H^{0}(X, d \mathcal{O})^{*}$ by the minimal closed complex sub- 
group containing the image of $H^{1}(X, \mathbb{Z})$ under the map

$$
H^{1}(X, \mathbb{Z}) \rightarrow H^{1}(X, \mathbb{C}) \rightarrow H^{0}(X, d \mathcal{O})^{*}
$$

(see $[\mathrm{R}]$ ). The Albanese map $\operatorname{Alb}: X \rightarrow \operatorname{Alb}(X)$ is give $\mathrm{n}$ by integration along paths starting at a fixed point. It has the functorial property tha $t$ any map from $X$ onto tori is factored through the Albanese map ([Ue75]). In $[\underline{\mathrm{R}}$ (section 2.1) the Albanese variety for a complex nilma nifold $M$ is described in terms of the space $\mathfrak{h}_{1}$ as

$$
\operatorname{Alb}(M)=\frac{H^{0}(X, d \mathcal{O})^{*} /\left(\mathfrak{h}_{1}\right)}{\operatorname{im}\left(H^{1}(X, \mathbb{Z}) \rightarrow H^{0}(X, d \mathcal{O})\right)^{*} / p\left(\mathfrak{h}_{1}\right)}=T,
$$

where $\mathfrak{h}_{\mathfrak{l}}$ is the same as in Corollary 1.11. Then we obtain that $T=\operatorname{Alb}(M)$ and

$$
a(M)=a(\operatorname{Alb}(M)) .
$$

\section{The averaging formalism}

Let $M=\Gamma \backslash G$ be a compact nilmanifold and $\nu$ a volume element on $M$ induced by a the Haar measure on the Lie group $G$ [Mi]. After a rescaling, we can suppose that $M$ has volume 1 . Notice that the Haar measure on $G$ is bi-invariant, because $G$ admits a lattice, and any Lie group admitting a lattice is unimodular.

Given any covariant $k$-tensor field $T: T M \times \ldots \times T M \rightarrow \mathcal{C}^{\infty}(M)$ on the nilmanifold $M$, one can define a covariant $k$-tensor

$$
T_{\text {inv }}: \mathfrak{g} \times \ldots \times \mathfrak{g} \rightarrow \mathbb{R}
$$

on the Lie algebra $\mathfrak{g}$ of $G$ by

$$
T_{i n v}\left(x_{1}, \ldots, x_{k}\right)=\int_{p \in M} T_{p}\left(\left.x_{1}\right|_{p}, \ldots,\left.x_{k}\right|_{p}\right) \nu
$$

for every $x_{1}, \ldots, x_{k} \in \mathfrak{g}$, where $\left.x_{l}\right|_{p}$ is the restriction of the left-invariant vector field $X_{l}$ to $p$. Clearly, $T_{i n v}=T$ for any tensor field $\mathrm{T}$ coming from a left-invariant one. In [B] it is shown that that if $\alpha$ is a differential $k$-form on $M$, then $(d \alpha)_{i n v}=d\left(\alpha_{i n v}\right)$. Moreover, $\left(\alpha_{i n v} \wedge \beta\right)_{i n v}=\alpha_{i n v} \wedge \beta_{i n v}$, for every differential forms $\alpha$ and $\beta$ on $M$.

We call the map Av: $\left(T^{*}\right)^{\otimes k} \longrightarrow\left(\mathfrak{g}^{*}\right)^{\otimes k}, \operatorname{Av}(T):=T_{i n v}$ averaging on a nilmanifold. The averaging defines a linear map $\tilde{\nu}: \Omega^{k}(M) \rightarrow \Lambda^{k} \mathfrak{g}^{*}$, given by $\tilde{\nu}(\alpha)=\alpha_{i n v}$ for every $k$-form $\alpha \in \Omega^{k}(M)$, which commutes with the differentials.

Moreover, by Nomizu theorem [N] $\tilde{\nu}$ induces an isomorphism $H^{k}(M) \rightarrow$ $H^{k}(\mathfrak{g})$ between the kth cohomology groups for every $k$. In particular, every 
closed $k$-form $\alpha$ on $\mathrm{M}$ is cohomologous to the invariant $k$-form $\alpha_{i n v}$ obtained by the averaging (see also $\overline{\mathrm{Ug}}]$ ) Indeed, by Nomizu Theorem $\alpha=\beta+d \gamma$, with $\beta$ invariant closed $k$-form. By using the averaging we have $\alpha_{i n v}=\beta+d \gamma_{i n v}$ and so $\alpha$ is cohomologous to $\alpha_{i n v}$.

\section{Positive currents on nilmanifolds}

\subsection{Holomorphic differentials}

Recall that holomorphic differentials on a complex manifold are closed, holomorphic 1-forms.

Definition 3.1: Let $M=\Gamma \backslash G$ be a nilmanifold. A differential form on $M$ is called invariant if its pullback to $M$ is invariant with respect to the left action of $G$ on itself.

Remark 3.2: Let $M=\Gamma \backslash G$ be a nilmanifold, $\mathfrak{g}$ the Lie algebra of $G$. Clearly, invariant differential forms are identified with $\Lambda^{*}(\mathfrak{g})$. Moreover, they are preserved by de Rham differential, which is identified with the Chevalley differential on $\Lambda^{*}(\mathfrak{g})$.

Proposition 3.3: Let $(M, I)$ be a complex nilmanifold, and $h$ a holomorphic differential. Then $h$ is an invariant differential form.

Proof: Let $\nu$ be a volume element on $M$ induced by a bi-invariant one on the Lie group $G$ such that $M$ has volume equal to 1 . A holomorphic differential $h$ is cohomologous to the invariant form $h_{\text {inv }}$ obtained by the averaging process. Since $I$ is invariant, $h_{i n v}$ has to be of type $(1,0)$ and thus $h=h_{\text {inv }}$. Indeed, closed $(1,0)$-forms cannot be exact, because they are holomorphic, hence (if exact) equal to differentials of a global holomorphic function.

Corollary 3.4: Let $M=\Gamma \backslash G$ be a complex nilmanifold, and $\mathfrak{g}$ its Lie algebra, and $\mathfrak{H}^{1}(M)$ the space of holomorphic differentials. Then

$$
\mathfrak{H}^{1}(M)=\left(\frac{\mathfrak{g} \otimes \mathbb{C}}{\mathfrak{g}^{1}+I\left(\mathfrak{g}^{1}\right)}\right)^{*},
$$

where $\mathfrak{g}^{1}=[\mathfrak{g}, \mathfrak{g}]$ denotes the commutator of $\mathfrak{g}$.

Proof: Let $h$ be a holomorphic differential. Since $h$ is invariant then it can be identified with an element of $(\mathfrak{g} \otimes \mathbb{C})^{*}$. Moreover, $h=\alpha+i I \alpha$, with 
$\alpha \in \mathfrak{g}^{*}, d \alpha=0$ and $d(I \alpha)=0$. By the conditions

$$
d \alpha(x, y)=-\alpha([x, y])=0, \quad d(I \alpha)(x, y)=\alpha(I[x, y])=0,
$$

for every $x, y \in \mathfrak{g}$, we get $\alpha\left(\mathfrak{g}^{1}\right)=\alpha\left(I \mathfrak{g}^{1}\right)=0$.

\subsection{Positive (1,1)-forms on a Lie algebra}

Throughout this subsection, we fix a nilpotent Lie algebra $\mathfrak{g}$ with a complex structure $I \in \operatorname{End}(\mathfrak{g})$ satisfying the integrability condition

$$
\left[\mathfrak{g}^{1,0}, \mathfrak{g}^{1,0}\right] \subset \mathfrak{g}^{1,0} .
$$

Definition 3.5: A semipositive Hermitian form on $(\mathfrak{g}, I)$ is a real form $\eta \in \Lambda^{2}\left(\mathfrak{g}^{*}\right)$ which is $I$-invariant (that is, of Hodge type $(1,1)$ ) and satisfies $\eta(x, I x) \geqslant 0$ for each $x \in \mathfrak{g}$. It is called positive definite Hermitian if this inequality is strict for all $x \neq 0$.

Definition 3.6: A subalgebra $\mathfrak{a} \subset \mathfrak{g}$ is called holomorphic if $I(\mathfrak{a})=\mathfrak{a}$ and $\left[\mathfrak{g}^{0,1}, \mathfrak{a}^{1,0}\right]^{1,0} \subset \mathfrak{a}^{1,0}$.

Claim 3.7: Let $\mathfrak{a} \subset \mathfrak{g}$ be a vector subspace, and $B:=\mathfrak{a} \cdot G$ the corresponding left-invariant sub-bundle in $T G$. Then

- $B$ is involutive (that is, Frobenius integrable) iff $\mathfrak{a}$ is a Lie subalgebra of $\mathfrak{g}$.

- $B$ is a holomorphic sub-bundle iff $\mathfrak{a}$ is a holomorphic subalgebra.

Proof: Let $x, y \in \mathfrak{a}$ and denote by the same letters the corresponding left-invariant vector fields. Clearly, $B$ is involutive if and only if $\mathfrak{a}$ is a Lie subalgebra of $\mathfrak{g}$. Similarly we have that $B$ is holomorphic if $[x+i I x, y-i I y] \epsilon$ $\mathfrak{a}^{1,0}$, for every $x \in \mathfrak{g}$ and $y \in \mathfrak{a}$.

Remark 3.8: Note that $V=\mathfrak{g}^{(1,0)}+\mathfrak{a}^{(0,1)}$ is involutive iff $\mathfrak{a}$ is holomorphic and $V+\bar{V}=\mathfrak{g}^{c}$. So $V$ is an "elliptic structure" in the terminology of [Jac], so by [Jac] it defines a holomorphic foliation.

We also note the obvious

Claim 3.9: If $V_{1}$ and $V_{2}$ are two elliptic structures in terminology of [Jac] on a complex manifold, containing the $(1,0)$ tangent bundle, then $V_{1} \cap V_{2}$ is also an elliptic structure. 
Definition 3.10: Let $\eta$ be a semipositive Hermitian form on $(\mathfrak{g}, I)$, and $N(\eta)$ the subspace in $\mathfrak{g}$ consisting of all vectors $x$ such that $\eta(x, I x)=0$. Then $N(\eta)$ is called the null-space of $\eta$.

In general we have the following

Claim 3.11: The nullspace

$$
N=\left\{x \in \mathfrak{g} \mid \iota_{x} \eta=0\right\}
$$

of a closed form $\eta \in \Lambda^{r} \mathfrak{g}^{*}$ is a Lie subalgebra of $\mathfrak{g}$.

Proof: Take $x, y \in N$ and arbitrary vectors $z_{1}, \ldots, z_{r-1} \in \mathfrak{g}$. Then, by Cartan's formula, $d \eta\left(x, y, z_{1}, \ldots, z_{r-1}\right)=\eta\left([x, y], z_{1}, \ldots z_{r-1}\right)=0$, since the rest of the terms vanish, because $x, y \in N$. Therefore $\eta\left([x, y], z_{1}, \ldots z_{r-1}\right)=0$ for any $z_{1}, \ldots, z_{r-1} \in \mathfrak{g}$, this means that $\iota_{[x, y]} \eta=0$, i.e $[x, y] \in N$.

Theorem 3.12: Let $\eta$ be a semipositive Hermitian form on $(\mathfrak{g}, I)$. Assume that its nullspace $N(\eta)$ is a holomorphic subalgebra. Then $N(\eta)$ contains $\mathfrak{g}^{1}+I \mathfrak{g}^{1}$, where $\mathfrak{g}^{1}=[\mathfrak{g}, \mathfrak{g}]$.

Proof: When the cohomology class of $\eta$ is rational, as happens in most applications, Theorem 3.12 has a simple proof. Since $[\eta]$ is rational, it can be represented by a rational form $\eta_{\mathbb{Q}} \in \Lambda^{2}(\mathfrak{g})$. Therefore, the leaves of $N(\eta)$ are rational Lie subalgebras in $\mathfrak{g}$. By Malcev's theorem, the leaves of $N(\eta)$ are compact. By construction, the leaf space $X$ of $N(\eta)$ is equipped with a transitive action by a nilpotent Lie group, hence it is a nilmanifold. Finally, $X$ inherits the complex structure from $X$, and $\eta$ defines a Kähler metric on $X$. However, a nilmanifold can be Kähler only if its fundamental group is abelian $([\mathrm{BG}])$. Therefore, $N\left(\eta_{\mathbb{Q}}\right)$ contains $[\mathfrak{g}, \mathfrak{g}]$.

For general $\eta$, Theorem 3.12 has a different (more complicated) proof.

Since $N(\eta)=\mathfrak{a}$ is holomorphic, we have

$$
[y+i I y, x-i I x]^{1,0} \in \mathfrak{a}^{1,0},
$$

for every $x \in \mathfrak{a}$ and for every $y \in \mathfrak{g}$. By a direct computation we obtain

$$
\begin{aligned}
{[y+i I y, x-i I x]^{1,0}=} & ([y, x]+[I y, I x]+I[I y, x]-I[y, I x]) \\
& -i I([y, x]+[I y, I x]+I[I y, x]-I[y, I x]) .
\end{aligned}
$$

Therefore, by the condition (3.1) we get

$$
[y, x]+[I y, I x]+I[I y, x]-I[y, I x] \in \mathfrak{a}, \quad \forall x \in \mathfrak{a}, \forall y \in \mathfrak{g} .
$$


By using the integrability condition

$$
[I y, I x]=[y, x]+I[I y, x]+I[y, I x]
$$

we have

$$
I[y, I x]=[I y, I x]-[y, x]-I[I y, x]
$$

and therefore the condition (3.2) becomes

$$
2([y, x]+I[I y, x]) \in \mathfrak{a}, \quad \forall x \in \mathfrak{a}, \forall y \in \mathfrak{g},
$$

i.e.

$$
\eta([y, x], z)=-\eta(I[I y, x], z), \quad \forall x \in \mathfrak{a}, \forall y, z \in \mathfrak{g} .
$$

Therefore

$$
\eta([y, x], I[y, x])=-\eta(I[I y, x], I[y, x])=-\eta([x, I y],[x, y]) .
$$

By $d \eta=0$, one gets

$$
\eta([x, y], w)=\eta([x, w], y),
$$

for every $x \in \mathfrak{a}, y, z \in \mathfrak{g}$. Thus

$$
\eta([x, I y],[x, y])=\eta\left(a d_{x}^{2}(y), I y\right), \quad \forall x \in \mathfrak{a}, \forall y \in \mathfrak{g}
$$

and consequently

$$
\eta([y, x], I[y, x])=-\eta\left(a d_{x}^{2}(y), I y\right), \quad \forall x \in \mathfrak{a}, \forall y \in \mathfrak{g} .
$$

By using (3.3), it is possible to show that $\mathfrak{a}$ is an ideal of $\mathfrak{g}$, i.e. that $[y, x] \in \mathfrak{a}$, for every $x \in \mathfrak{a}$ and $y \in \mathfrak{g}$.

Since $\eta$ is a semipositive $(1,1)$-form and $\mathfrak{a}$ is its null-space, the relation $\eta([y, x], I[y, x])=0$ implies that $[x, y] \in \mathfrak{a}$. Therefore, by (3.3), in order to prove that $[y, x] \in \mathfrak{a}$, for every $x \in \mathfrak{a}$ and for every $y \in \mathfrak{g}$, it is sufficient to show that $[x,[x, y]] \in \mathfrak{a}$ for any $x \in \mathfrak{a}$. This would follow if we prove that

$$
\left[\mathfrak{a}, \mathfrak{g}^{1}\right] \subset \mathfrak{a} .
$$

Since $\mathfrak{g}$ is nilpotent there exists $s$ such that $\mathfrak{g}^{s}=\{0\}$ and $\mathfrak{g}^{s-1} \neq\{0\}$ and we have the descending series of ideals

$$
\mathfrak{g}=\mathfrak{g}^{0} \supset \mathfrak{g}^{1} \supset \ldots \supset \mathfrak{g}^{i} \supset \mathfrak{g}^{i+1} \supset \ldots \supset \mathfrak{g}^{s-1} \supset \mathfrak{g}^{s}=\{0\} .
$$

Now we can prove that $\left[\mathfrak{a}, \mathfrak{g}^{1}\right] \subset \mathfrak{a}$ by induction on $i$ in the following way: by using (3.3) we can show that

(A) if the condition $\left[\mathfrak{a}, \mathfrak{g}^{i+1}\right] \subset \mathfrak{a}$ holds, then the condition $\left[\mathfrak{a}, \mathfrak{g}^{i}\right] \subset \mathfrak{a}$ holds. 
Since $\mathfrak{g}$ is nilpotent there exists $s$ such that $\mathfrak{g}^{s}=\{0\}$ and $\mathfrak{g}^{s-1} \neq\{0\}$.

At the first step $i=s-1$ we have that (A) holds. So by induction we obtain that (A) holds for $i=0$. Consequently, $\mathfrak{a}=N(\eta)$ is an ideal of $\mathfrak{g}$ and $\eta$ induces a Kähler form on the nilpotent Lie algebra $\mathfrak{g} / \mathfrak{a}$. By $[\mathrm{BG}$, the Kähler nilpotent Lie algebra $\mathfrak{g} / \mathfrak{a}$ has to be abelian. Therefore $\mathfrak{g}^{1} \subset \mathfrak{a}$. Since $\eta$ is $(1,1)$-form, its null-space $\mathfrak{a}$ is $I$-invariant, hence $\mathfrak{a}$ contains $\mathfrak{g}^{1}+I \mathfrak{g}^{1}$.

\subsection{Averaging the positive currents}

The following result directly follows from the averaging.

Proposition 3.13: Let $M=\Gamma \backslash G$ be a compact quotient of a unimodular Lie group $G$ by a lattice $\Gamma$ and $I$ the complex structure on $M$ obtained from an invariant complex structure on $G$. Let $T_{\eta}$ be the positive, closed (1,1)-current induced by the algebraic reduction $\varphi: M \rightarrow X$ from some Kähler form $\eta$ on $X$ (Definition 1.5). If $\operatorname{Av}(T)$ is its average, then $\operatorname{Av}(T)$ is a semipositive, closed, $G$-invariant differential form, and its rank is no less than the rank of the absolutely continuous part of $T_{\eta}$.

Proof: If $X$ and $Y$ are left-invariant vector fields on $M$, then $T_{\eta}(X, Y)$ is a measurable function when we consider $T_{\eta}$ as a form with distributional coefficients in local coordinates. So $\operatorname{Av}(T)$ is well defined as in Section 2. Then $\operatorname{Av}(T)$ is a closed invariant form of type $(1,1)$ and the only thing to check is the statement about its rank. By the definition it follows that $\operatorname{Av}(T)(X, I X)=0 \Leftrightarrow T_{p}\left(\left.X\right|_{p},\left.I X\right|_{p}\right)=0$ for almost all $p \in M$. So $X$ is in the kernel of $\operatorname{Av}(T)$ only if it is in the kernel of $T_{p}$ for almost all $p$.

Remark 3.14: As a corollary we obtain that if such space admits a Kähler current, it is Kähler. In particular from [DP] it follows that such spaces are never in Fujiki's class $\mathcal{C}$. Note that the proof of this fact in [DP] uses also the Kähler current arising from the pull-back of a Kähler form.

Proposition 3.15: Let $T$ be a positive, closed $(1,1)$-current on a nilmanifold $M=G / \Gamma$, and $\mathcal{F}$ the null-space foliation of its absolutely continuous part. Then the sub-bunlde associated with $\mathcal{F}$ contains a homogeneous sub-bundle $\Sigma$ obtained by left translates of $\mathfrak{g}^{1}+I \mathfrak{g}^{1}$, where $\mathfrak{g}^{1}=[\mathfrak{g}, \mathfrak{g}]$, and $\mathfrak{g}$ is the Lie algebra of $G$.

Proof: Let $A v$ be the averaging map defined in Section 2. The nullspace of the form $\operatorname{Av}(T)$ is contained in the intersection of all left translates of $\mathcal{F}$, hence by Claim 3.9 it is also holomorphic. Then Theorem 3.12 implies that $N(\operatorname{Av}(T))$ contains $\mathfrak{g}^{1}+I \mathfrak{g}^{1}$. 
Proof of Theorem 1.1; Let $M \longrightarrow X$ be the algebraic reduction map (Definition 1.4), and $\eta$ the pullback of the Kähler form on $X$. Averaging (Proposition 3.13) transforms $\eta$ into an invariant, closed, semipositive form. Then $\eta$ vanishes on $\Sigma$ by Proposition 3.15.

Proof of Theorem 1.8: Now we can prove Theorem 1.8, Let $M=$ $\Gamma \backslash G$ be a nilmanifold, and $\varphi: M \rightarrow X$ the algebraic reduction map. The pullback $\varphi^{*} \omega_{X}$ of a Kähler form $\omega_{X}$ is a current $T$ on $M$ (Definition 1.5). By Proposition 3.15, the rank of its absolutely continuous part is no greater that

$$
\operatorname{dim} \frac{\mathfrak{g}}{\mathfrak{g}^{1}+I \mathfrak{g}^{1}}=\operatorname{dim} \mathfrak{H}^{1}(M)
$$

(the latter equality follows from Corollary 3.4).

\section{Examples}

All 2-dimensional compact complex nilmanifolds are classified and correspond to tori and primary Kodaira surfaces. Their algebraic dimension is known. In this section we'll consider the algebraic dimension of the complex nilmanifolds in dimension 3 and note that for other complex homogeneous spaces the inequality $a(M) \leqslant \mathfrak{H}^{1}(M)$ may not hold.

Many nilmanifolds admit holomorphic fibrations and we'll need the following:

Remark 4.1: In general (see [Ue75, Theorem 3.8]), if a complex manifold $M$ is the total space of a holomorphic fibration $\pi: M \rightarrow B$ we always have the inequality

$$
a(M) \geqslant a(B) .
$$

\subsection{Algebraic dimension of complex 2-tori}

Following $[\mathrm{BL}$ we have the following description of the algebraic dimension of the complex 2-tori.

Let $T^{4}$ be the tori defined as $\mathbb{R}^{4} / \mathbb{Z}^{4}$ where $\mathbb{Z}^{4}$ is the standard lattice in $\mathbb{R}^{4}$. Let $J \in \operatorname{End}\left(\mathbb{R}^{4}\right), J=\left(\begin{array}{cc}A & B \\ C & D\end{array}\right)$ be a complex structure with $A, B, C, D$ $2 \times 2$-blocks and $B$ nondegenerate. From BL (p.10) we can identify what is the period lattice of the complex tori with structure $J$. If $X=\mathbb{C}^{2} /\left(\tau, I d_{2}\right) \mathbb{Z}^{4}$ is a complex tori defined by a complex $2 \times 2$ matrix $\tau$, then the complex 
structure $J_{\tau}$ on $T^{4}$ such that $X \cong\left(T^{4}, J_{\tau}\right)$ as complex manifold is given by

$$
J_{\tau}=\left(\begin{array}{lc}
y^{-1} x & y^{-1} \\
-y-x y^{-1} x & -x y^{-1}
\end{array}\right),
$$

where $x$ and $y$ are the real and imaginary parts of $\tau$. Reversing the construction gives that for $J$ as above,

$$
\tau_{J}=B^{-1} A+i B^{-1} .
$$

We also need the relation to complex structures, defined in terms of a basis of $(1,0)$-forms. If $J_{0}$ is a fixed complex structure and $\omega_{j}=e_{j}+\sqrt{-1} J_{0} e_{j}, j=1,2$ is a basis of (linear) $(1,0)$-forms for $J_{0}$, we define another complex structure $J$ as

$$
\begin{aligned}
& \alpha_{1}=\omega_{1}+a \overline{\omega_{1}}+b \overline{\omega_{2}} \\
& \alpha_{2}=\omega_{2}+c \overline{\omega_{1}}+d \overline{\omega_{2}}
\end{aligned}
$$

being the basis of $(1,0)$-forms of $J$. If $X=\left(\begin{array}{ll}a & b \\ c & d\end{array}\right)=X_{1}+i X_{2}$, then the relation between $X$ and the matrix representing $J$ in the basis $\left(e_{i}, J_{0} e_{i}\right)$ is given by

$$
J=\left(\begin{array}{lc}
I d+X_{1} & X_{2} \\
X_{2} & I d-X_{1}
\end{array}\right)^{-1}\left(\begin{array}{lc}
0 & I d \\
-I d & 0
\end{array}\right)\left(\begin{array}{lc}
I d+X_{1} & X_{2} \\
X_{2} & I d-X_{1}
\end{array}\right)
$$

We'll use the explicit form of $J$ and $\tau_{J}$ when $X_{1}=\left(\begin{array}{cc}0 & a \\ 0 & 0\end{array}\right)$ and $X_{2}=$ $\left(\begin{array}{ll}0 & b \\ 0 & 0\end{array}\right)$. Direct calculation (using the fact that $X_{1}^{2}=X_{2}^{2}=X_{1} X_{2}=X_{2} X_{1}=$ $0)$ gives $J=\left(\begin{array}{lc}2 X_{2} & I d-2 X_{1} \\ -I d-2 X_{1} & -2 X_{2}\end{array}\right)$ and

$$
\tau_{J}=\left(\begin{array}{cc}
i & 2 a+2 b i \\
0 & i
\end{array}\right)=i I D+2 X
$$

To determine the algebraic dimension of $\left(T^{4}, J\right)$, we need first the NeronSeveri group $N S(J)$ of $J$. Let $\tau_{i j}$ are the components of $\tau_{J}$ and

$$
E=\left(\begin{array}{llll}
0 & a & b & c \\
-a & 0 & d & e \\
-b & -d & 0 & f \\
-c & -e & -f & 0
\end{array}\right) \in M_{4}(\mathbb{Z})
$$

be an integral matrix. Then $N S(J)=\left\{E \in M_{4}(Z) \mid a+d \tau_{11}-b \tau_{12}+f \tau_{21}-\right.$ $\left.c \tau_{22}+\operatorname{edet}(\tau)=0\right\}$. With these notations in mind, the algebraic dimension of $\left(T^{4}, J\right)$ is determined by 


$$
a(J)=\frac{1}{2} \max \left\{\operatorname{rank}\left(J^{T} E\right) \mid E \in N S(J), J^{T} E \geqslant 0\right\},
$$

where the superscript $T$ means transposition. Note that not all complex structures are described in this way - we have the non-degeneracy condition on $B$ which is required for $\left(\tau, I d_{2}\right)$ to be a period matrix. It is well known that $a(J)$ could be any of 0,1 or 2 .

The integrality condition leads to the fact that generically $a(J)=0$. For $a(J) \geqslant 0$ from $\mathrm{BL}$ p.59, we know that $a(J)=1$ exactly when the torus admits a period matrix $\left(\tau, I d_{2}\right)$ with

$$
\tau=\left(\begin{array}{cc}
\tau_{1} & \alpha \\
0 & \tau_{2}
\end{array}\right)
$$

with

$$
\alpha \notin\left(\tau_{1}, 1\right) M_{2}(\mathbb{Q})\left(\begin{array}{l}
1 \\
\tau_{2}
\end{array}\right)
$$

where $M_{2}(\mathbb{Q})$ is the set of $2 \times 2$-matrices. In particular in (4.1), when $X=$ $\left(\begin{array}{cc}0 & \sqrt{2}-i \sqrt{3} \\ 0 & 0\end{array}\right)$, the algebraic dimension of $\left(T^{4}, J\right)$ is one.

\subsection{3-dimensional complex nilmanifolds}

Definition 4.2: Let $\mathfrak{g}$ be a nilpotent Lie algebra. A rational structure for $\mathfrak{g}$ is a subalgebra $\mathfrak{g}_{\mathbb{Q}}$ defined over $\mathbb{Q}$ such that $\mathfrak{g} \cong \mathfrak{g}_{\mathbb{Q}} \otimes \mathbb{R}$. A subalgebra $\mathfrak{h}$ of $\mathfrak{g}$ is said to be rational with respect to a given rational structure $\mathfrak{g}_{\mathbb{Q}}$ if $\mathfrak{h}_{\mathbb{Q}}:=\mathfrak{h} \mathfrak{g}_{\mathbb{Q}}$ is a rational structure for $\mathfrak{h}$.

It follows from a result of Malcev $\mathrm{M}$ ] that $\Gamma \backslash G$ is compact, where $\mathrm{G}$ is a simply connected $\mathrm{k}$-step nilpotent Lie group admitting a basis of left invariant 1-forms for which the coefficients in the structure equations are rational numbers, and $\Gamma$ is a lattice in $G$ of maximal rank (i.e., a discrete uniform subgroup, cf. $[\mathrm{Rag}]$ ). Such a lattice $\Gamma$ exists in $G$ if and only if the Lie algebra $\mathfrak{g}$ of $G$ has a rational structure. Indeed, If $\Gamma$ is a lattice of $G$ then its associated rational structure is given by the $\mathbb{Q}$-span of $\log \Gamma$.

An invariant complex structure $J$ on a nilmanifold $\Gamma \backslash G$ is called rational if it is compatible with the rational structure of $G$, i.e. $J\left(\mathfrak{g}_{\mathbb{Q}}\right) \subseteq \mathfrak{g}_{\mathbb{Q}}$.

Remark 4.3: Let $M=\Gamma \backslash G$ be a complex nilmanifold of complex dimension $n$ endowed with an invariant rational complex structure $J$. Consider the surjective homomorphism $\mathfrak{g} \rightarrow \mathfrak{g} / \mathfrak{g}_{J}^{1}$, where $\mathfrak{g}_{J}^{1}=\mathfrak{g}^{1}+J \mathfrak{g}^{1}$. Let $G, G_{J}^{1}$ and $K$ be the simply connected Lie groups respectively with Lie algebra $\mathfrak{g}, \mathfrak{g}_{J}^{1}$ and $\mathfrak{g} / \mathfrak{g}_{J}^{1}$, then we have the surjective homomorphism

$$
\begin{gathered}
p: G \rightarrow K, \\
-14-
\end{gathered}
$$

VERSION 2.0, APRIL 8, 2016 
with $K$ abelian. Since $J$ is rational then, by [CF $\mathfrak{g}_{J}^{1}$ is a rational subalgebra of $\mathfrak{g}$. Then $\Gamma^{1}:=\Gamma \cap G_{J}^{1}$ is a uniform discrete subgroup of $G_{J}^{1}$ [CG], Theorem 5.1.11. By [CG, Lemma 5.1.4 (a)], $p(\Gamma)$ is a uniform discrete subgroup of $K$ (i.e. $p(\Gamma) \backslash K$ ) is compact, cf. $\mathrm{Rag}$ ). By Lemma 2 in $[\mathrm{CF}$ the map

$$
\tilde{p}: \Gamma \backslash G \rightarrow p(\Gamma) \backslash K
$$

is a holomorphic fibre bundle. Moreover, since $K$ is abelian, $p(\Gamma) \backslash K$ is a complex torus $\mathbb{T}$ of complex dimension $\mathfrak{H}^{1}(M)=n-\operatorname{dim}_{\mathbb{C}} \mathfrak{g}_{J}^{1}$. Therefore, if $\mathfrak{H}^{1}(M)=1$, the torus $\mathbb{T}$ is algebraic, and by Remark 4.1 and Theorem 1.8 we have $a(M)=1$.

Remark 4.4: Let $M=\Gamma \backslash G$ be a complex nilmanifold of complex dimension $n$ endowed with an invariant complex structure $J$ such that $J \mathfrak{g}^{1}=\mathfrak{g}^{1}$, then $M$ is the total space of a holomorphic fibration $\pi: M \rightarrow \mathbb{T}$, with $\mathbb{T}$ a complex torus of complex dimension $\mathfrak{H}^{1}(M)=n$ - $\operatorname{dim}_{\mathbb{C}} \mathfrak{g}^{1}$. Therefore, if $\mathbb{T}$ is algebraic, i.e. $a(\mathbb{T})=\mathfrak{H}^{1}(M)$, we have by Remark 4.1 and Theorem 1.8 we have $\mathfrak{a}(B)=$ $\mathfrak{a}(M)=\mathfrak{H}^{1}(M)$.

Note that if $J$ is bi-invariant, i.e. if $M=\Gamma \backslash G$ is complex parallelalizable then $J \mathfrak{g}^{1}=\mathfrak{g}^{1}$. For a general result on the algebraic dimension of complex parallelalizable solvmanifolds see Theorem 2 and its Corollary in [Sak].

We will apply the previous remarks to complex nilmanifolds of complex dimension 3.

Example 4.5: Let $J$ be a complex structure on a real 6-dimensional nilpotent Lie algebra. For the notion of "nilpotent complex structure" on a nilmanifold, please see $\mathrm{Ug}$. By $\mathrm{Ug}$ the complex structure $J$ is either nilpotent or non-nilpotent and

(a) If $J$ is non nilpotent, then there is a basis of $(1,0)$-forms $\left(\omega^{1}, \omega^{2}, \omega^{3}\right)$ such that

$$
\left\{\begin{array}{l}
d \omega^{1}=0 \\
d \omega^{2}=E \omega^{1} \wedge \omega^{3}+\omega^{1} \wedge \bar{\omega}^{3}, \\
d \omega^{3}=A \omega^{1} \wedge \bar{\omega}^{1}+i b \omega^{1} \wedge \bar{\omega}^{2}-i b \bar{E} \omega^{2} \wedge \bar{\omega}^{1},
\end{array}\right.
$$

where $A, E \in \mathbb{C}$ with $|E|=1$ and $b \in \mathbb{R}-\{0\}$.

(b) If $J$ is nilpotent, then there is a basis of $(1,0)$-forms $\left(\omega^{1}, \omega^{2}, \omega^{3}\right)$ sat- 
isfying

$$
\left\{\begin{aligned}
d \omega^{1}= & 0 \\
d \omega^{2}= & \varepsilon \omega^{1} \wedge \bar{\omega}^{1} \\
d \omega^{3}= & \rho \omega^{1} \wedge \omega^{2}+(1-\varepsilon) A \omega^{1} \wedge \bar{\omega}^{1}+B \omega^{1} \wedge \bar{\omega}^{2} \\
& +C \omega^{2} \wedge \bar{\omega}^{1}+(1-\varepsilon) D \omega^{2} \wedge \bar{\omega}^{2}
\end{aligned}\right.
$$

where $A, B, C, D \in \mathbb{C}$ and $\varepsilon, \rho \in\{0,1\}$.

Suppose that the real and imaginary parts of the complex structure equations constants are rational, then $G$ admits a lattice $\Gamma$. Let $M=\Gamma \backslash G$ be the compact quotient endowed with the induced invariant complex structure $J$.

In the case $(a)$ we have that $\mathfrak{H}^{1}(M)=1$. In the case $(b)$ we have the following cases:

(b1) $\mathfrak{H}^{1}(M)=1$ if $\varepsilon=1$ and $\rho^{2}+|B|^{2}+|C|^{2} \neq 0$

(b2) $\mathfrak{H}^{1}(M)=2$ if $\varepsilon=0$

(b3) $\mathfrak{H}^{1}(M)=2$ if if $\varepsilon=1$ and $\rho=B=C=0$

Therefore, in the cases $(a)$ and $(b 1)$, since $J$ is rational, by Theorem 1.8 and previous remarks, we have $a(M)=\mathfrak{H}^{1}(M)=1$. In the case $(b 3), G$ is the direct product of the real 3-dimensional Heisenberg group by $\mathbb{R}^{3}$.

In the case $(b 2), \rho^{2}+|B|^{2}+|C|^{2}+\left|D^{2}\right|=0$, then $G$ is the direct product of the real 3-dimensional Heisenberg group by $\mathbb{R}^{3}$. If $\rho^{2}+|B|^{2}+|C|^{2}+\left|D^{2}\right| \neq 0$, then $J \mathfrak{g}^{1}=\mathfrak{g}^{1}$ is a rational subalgebra of complex dimension 1 and $M$ is the total space of a holomorphic fibre bundle over a complex torus $\mathbb{T}$ of complex dimension 2 . Therefore, if $\mathbb{T}$ is algebraic then by previous remarks we have $a(M)=\mathfrak{H}^{1}(M)=2$.

An explicit example of the case (b2) is given by the well known Iwasawa manifold $M$. The Iwasawa manifold $M$ is defined as the quotient $\Gamma \backslash G$, where

$$
G=\left\{\left(\begin{array}{ccc}
1 & z_{1} & z_{3} \\
0 & 1 & z_{2} \\
0 & 0 & 1
\end{array}\right) \mid z_{i} \in \mathbb{C}\right\}
$$

is the complex Heisenberg group and $\Gamma$ is the lattice defined by taking $z_{i}$ to be Gaussian integers, acting by left multiplication. The 1-forms

$$
\omega^{1}=d z_{1}, \omega^{2}=d z_{2}, \omega^{3}=-d z_{3}+z_{1} d z_{2}
$$

are left-invariant on $G$. Define a $r$ basis $\left(e^{1}, \ldots, e^{6}\right)$ of real 1 -forms by setting

$$
\omega^{1}=e^{i}+i e^{2}, \omega^{2}=e^{3}+i e^{4}, \omega^{6}=e^{5}+i e^{6} .
$$


These 1-forms are pullbacks of corresponding 1-forms on the compact quotient $M$, which we denote by the same symbols and they satisfy the structure equations

$$
\begin{aligned}
& d e^{j}=0, j=1,2,3,4, \\
& d e^{5}=e^{1} \wedge e^{3}-e^{2} \wedge e^{4}, \\
& d e^{6}=e^{1} \wedge e^{4}+e^{2} \wedge e^{3} .
\end{aligned}
$$

The Iwasawa manifold $M$ is the total space of a principal $T^{2}$-bundle over the real torus $T^{4}$. The mapping $p: M \rightarrow T^{4}$ is induced from the projection $\left(z_{1}, z_{2}, z_{3}\right) \mapsto\left(z_{1}, z_{2}\right)$ and the space of invariant 1-forms annihilating the fibres of $\mathrm{p}$ is given by $\operatorname{span}\left\langle e^{1}, e^{2}, e^{3}, e^{4}\right\rangle$

Then $p: M \rightarrow T^{4}$ induces a complex structure $\hat{J}$ on the real 4-dimensional torus $T^{4}$ such that $p:(M, J) \rightarrow\left(T^{4}, \hat{J}\right)$ is holomorphic.

Claim 4.6: For the invariant complex structures on the Iwasawa manifold, $M, a(M)=a\left(T^{4}, \hat{J}\right)$

Proof: From Theorem 1.1, any meromorphic function is constant on the fibers of the projection $M \longrightarrow\left(T^{4}, \hat{J}\right)$. This implies that $a(M)=a\left(T^{4}, \hat{J}\right)$.

Now consider the possible algebraic dimension of $M$. First note that not all complex structures on the base $T^{4}$ arise as $\hat{J}$. A description of the set of such $J$ is given in $[\mathrm{KS}]$ and it is known that it has 4 components. We'll use one of them to see that that invariant structures on the Iwasawa nilmanifold can have algebraic dimension 0,1 and 2 . If we start with the canonical structure on $T^{4}$ which corresponds to the standard lattice in $R^{4}$ it gives the case of algebraic dimension 2. From [KS] formula (10) we know that the matrix $X=\left(\begin{array}{ll}0 & \sqrt{2}-i \sqrt{3} \\ 0 & 0\end{array}\right)$ corresponds to a complex structure on $T^{4}$ which is $\hat{J}$ for some $J$ on $M$. On the other side, the period matrix $\tau$, as explained above is $i I d+2 X$ and has $\tau=x+i y$ with $x=\left(\begin{array}{ll}0 & 2 \sqrt{2} \\ 0 & 0\end{array}\right)$ and $y=\left(\begin{array}{ll}1 & -2 \sqrt{3} \\ 0 & 1\end{array}\right)$. So for such $\tau$ the algebraic dimension of the base is 1. In particular we have strict inequalities in Theorem 1.8, Again, the base generically has algebraic dimension 0 , which leads to vanishing of the algebraic dimension of $J$ on the Iwasawa nilmanifold.

Example (Compact Lie groups) It is well known that every even-dimensional compact Lie group $G$ admits an invariant complex structure ([Sam]). The construction uses the structure theory for semisimple Lie algebras and provides a holomorphic fibration $G \rightarrow F l$ to the complete flag manifold 
$F l=G / T$, where $T$ is a maximal torus in $G$. The manifold $F l$ is algebraic and its algebraic dimension is equal to its complex dimension. On the other side, $\mathfrak{g}^{s s}=\mathfrak{g}^{\prime}$, so the space of holomorphic differentials is trivial. Hence by Remark 4.1, $a(M) \geqslant \operatorname{dim}(F l)>\mathfrak{H}^{1}(M)=0$. Similarly, for non-Kähler compact complex homogeneous spaces $G / H$ with $G$ compact, the inequality $a(M) \leqslant \mathfrak{H}^{1}(M)$ does not hold in general.

Acknowledgements: The work on this project started when the first two authors visited the HSE in Moscow. They are grateful for the hospitality and stimulating environment at the "Bogomolov Lab" there. Part of the work was done while the second named author visited University di Torino (Torino), Max Plank Institute for Mathematics (Bonn) and Institute of Mathematics at the Bulgarian Academy of Sciences. He thanks all institutions for the hospitality. The visits were partially funded by grants from G.N.S.A.G.A. and MPIM. The third named author is thankful to Alexandra Victorova and Alexandra Skripchenko for their ideas and many stimulating discussions of the subject. We also thank S. Rollenske for his useful comments on the paper.

\section{References}

[BD] M.L. Barberis, I. Dotti, Abelian complex structures on solvable Lie algebras, J. Lie Theory 14(1) (2004), 25-34.

[BDV] M. L. Barberis, I. G. Dotti, M. Verbitsky, Canonical bundles of complex nilmanifolds, with applications to hypercomplex geometry, Math. Res. Lett. 16 (2009), 331-347.

[B] F. Belgun, On the metric structure of non-Kähler complex surfaces, Math. Ann. 317 (2000), 1-40.

[BG] C. Benson, C.S. Gordon, Kähler and symplectic structures on nilmanifolds, Topology 27(4) (1988) 513-518.

[BL] C. Birkenhacke, H. Lange, Complex Tori, Progress in Mathematics 177, Birkhauser, 1999.

[Bouck] S. Boucksom. On the volume of a line bundle Intern. J. Math. 13, no 10 (2002) $1043-1063$.

[Ca81] F. Campana, Coréduction algébrique d'un espace analytique faiblement kählérien compact, Invent. Math. 63 (1981) 187-223.

$[\mathrm{CF}]$ S. Console, A. Fino, Dolbeault cohomology of compact nilmanifolds, Transformation Groups 6 (2) (2001), 111-124.

[CFP] S. Console, A. Fino, Y.S. Poon, Stability of abelian complex structures, Internat. J. Math. 17 (2006), no. 4, 401-416. 
[CFG1] L.A. Cordero, M. Fernández, A. Gray, Symplectic manifolds with no Kähler structure, Topology 25 (1986), no. 3, 375-380.

[CFG2] L.A. Cordero, M. Fernández, A. Gray, The Frölicher spectral sequence and complex compact nilmanifolds, C. R. Acad. Sci. Paris Sér. I Math. 305 (1987), no. 17, 753-756.

[CFL] L.A. Cordero, M. Fernández, M. de León, Examples of compact complex manifolds with no Kähler structure, Portugal. Math. 44 (1987), no. 1, 4962.

[CFGU] L.A. Cordero, M. Fernández, A. Gray, L. Ugarte, Compact nilmanifolds with nilpotent complex structures: Dolbeault cohomology, Trans. Amer. Math. Soc. 352 (12), 5405-5433.

[CG] L.J. Corwin, F.P. Greenleaf, Representations of nilpotent Lie groups and their applications, Cambridge studies in advanced mathematics 18, Cambridge, New York, 1990.

[DP] J.-P. Demailly, M. Paun, Numerical characterization of the Kähler cone of a compact Kähler manifold, Annals of Math. 159 (2004) 1247-1274.

[DF1] I. Dotti, A. Fino, Abelian hypercomplex 8-dimensional nilmanifolds, Ann. Global Anal. Geom. 18 (1) (2000), 47-59.

[FG] M. Fernández, A. Gray, The Iwasawa manifold, Differential geometry, Peñíscola 1985, 157-159, Lecture Notes in Math., 1209, Springer, Berlin, 1986.

[FG] A. Fino, G. Grantcharov, On some properties of the manifolds with skewsymmetric torsion and holonomy $S U(n)$ and $S p(n)$, Adv. Math. 189 (2004), no. $2,439-450$.

[G] H. Guggenheimer, Sur les variétés qui possèdent une forme extérieure quadratique fermée, C. R. Acad. Sci. Paris 232, (1951). 470-472.

[H] K. Hasegawa, Minimal models of nilmanifolds, Proc. Amer. Math. Soc. 106 (1989), no. $1,65-71$.

[Jac] H. Jacobowitz, Transversely holomorphic foliations and CR structures. VI Workshop on Partial Differential Equations, Part I (Rio de Janeiro, 1999). Mat. Contemp. 18 (2000), 175-194.

[KS] G. Ketsetzis, S. Salamon, Complex structures on the Iwasawa manifold, Adv. Geom. 4 (2004), no. 2, 165-179.

[M] A. I. Mal'čev, On a class of homogeneous spaces, AMS Translation No. 39 (1951).

[Mi] J. Milnor, Curvatures of left invariant metrics on Lie groups, Adv. Math. 21 (1976), 293-329.

[N] K. Nomizu, On the cohomology of compact homogeneous spaces of nilpotent Lie groups, Ann. of Math. 59 (1954), 531-538. 
[Pet] Th. Peternell, Modifications, Several complex variables, VII, 285-317, Encyclopaedia Math. Sci., 74, Springer, Berlin, (1994)

[Rag] M. S. Raghunathan, Discrete subgroups of Lie groups, Springer E.M.G. 68, Berlin, Heidelberg, New York, 1972.

[R] S. Rollenske, Geometry of nilmanifolds with left-invariant complex structure and deformations in the large, Proc. Lond. Math. Soc. (3) 99 (2009), no. 2, 425-460.

[Sak] Y. Sakane, On compact complex parallelalisable solvmanifolds, Osaka J. Math. 13 (1976), 187-212.

[S] S.M. Salamon, Complex structures on nilpotent Lie algebras, J. Pure Appl. Algebra 157 (2001), 311-333.

[Sam] H. Samelson, A class of compact-analytic manifolds, Portugaliae Math. 12 (1953), 129-132.

[T] W. P. Thurston, Some simple examples of symplectic manifolds, Proc. Amer. Math. Soc. 55 (1976), no. 2, 467-468.

[Ue75] K. Ueno, Classification theory of algebraic varieties and compact complex spaces, Lecture Notes in Mathematics 439, Springer-Verlag, Berlin-New York (1975).

[Ug] L. Ugarte, Hermitian structures on six-dimensional nilmanifolds, Transform. Groups 12 (2007), 175-202.

[W] J. Winkelmann, Complex Analytic Geometry of Complex Parallelizable Manifolds, Mem. Soc. Math. France (N.S.) 72-73, Soc. Math. France, Montrouge, 1998.

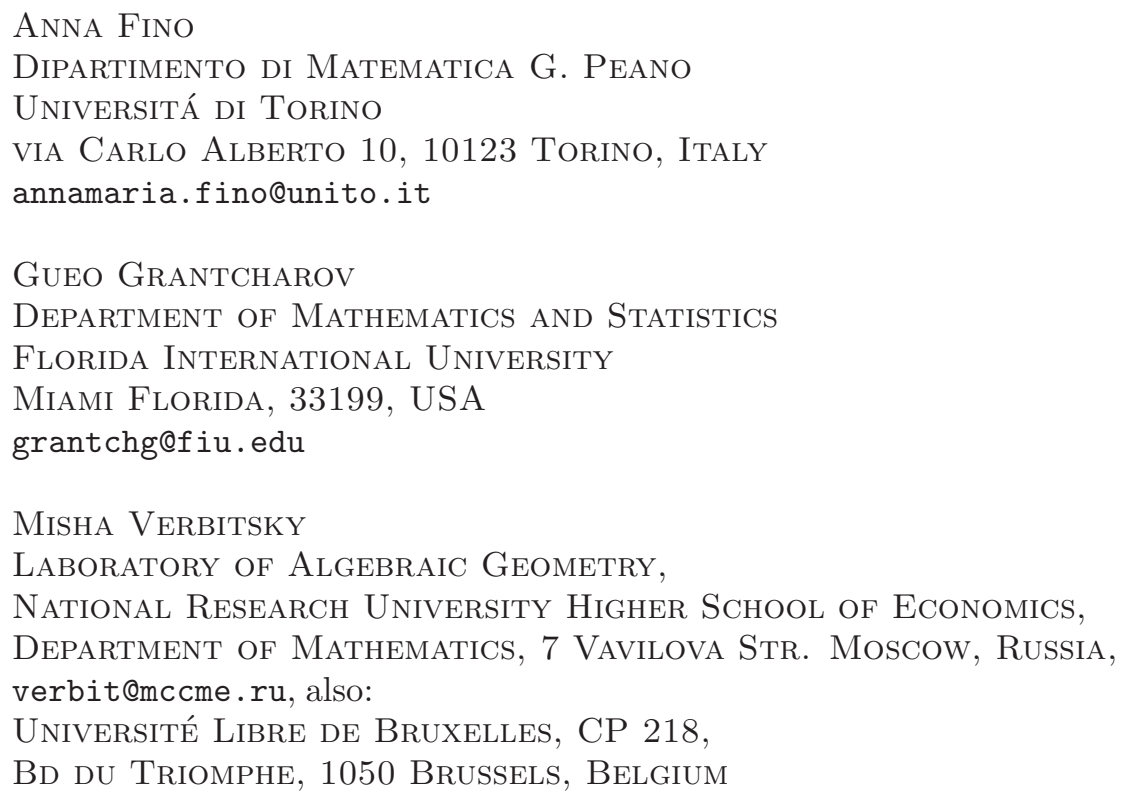

